\title{
Impact of the Internet/World-Wide Web on today's society with reference to business
}

\author{
ANM Paterson \\ Post Graduate Diploma in Infomration Management \\ anmpaterson@beauty.hscr.ac.za
}

\section{Contents}

Introduction

Value of the Internet/Web to business

New economic rules of the Internet/Web

Analysis of growth of e-commerce

Internet/Web as a site which creates new needs and opportunities for new business forms Internet/Web business, the state and the legal infrastructure

Conclusion

References

\section{Introduction}

The juxtaposition of the words Internet/World-Wide Web in the title of this article implies equivalence between the Internet and the Web. What distinguishes the Web from the Internet? The Webster Dictionary (2000) defines the World-Wide Web as 'a part of the Internet designed to allow easier navigation of the network through the use of graphical user interfaces and hypertext links between different addresses [called also Web]'. The Webster definition of the Internet is 'an electronic communications network that connects computer networks and organizational computer facilities around the world'. The Instantweb Computing Dictionary(2000) defines the Web as: 'An Internet client-server hypertext distributed information retrieval system ... On the WWW everything (documents, menus, indices) is represented to the user as a hypertext object in HTML format. Hypertext links refer to other documents by their URLs. These can refer to local or remote resources accessible via FTP, Gopher, Telnet or news, as well as those available via the http protocol used to transfer hypertext documents'. The Instantweb Dictionary defines internet as: 'Any set of networks interconnected with routers. The Internet is the biggest example of an internet.'

The definitions of the Internet given by both sources are broader than the definitions of the Web. The definitions of the Web focus on the technological medium of HTML through which the Web is constructed, whereas the definitions of the Internet focus on the multiplicity of linkages between computer networks. Either way, the aim of this article is to examine how the combined resources of the Web/Internet have either become integrally necessary to many functions of business, or are fundamentally changing the nature of 
business processes across a wide range of economic sectors.

The strategic influence of the Web/Internet on business derives from the fact that it serves as a medium that practically did not exist until the early 1990s. A number of technological innovations had to be in place before the Internet/Web could become viable as a new market place. These included: the writing of modem programmes to enable computers to talk over phone lines, the adoption of protocols (e.g. TCP-IP), the introduction of a domain name system, Berners-Lee's invention of HTML, and the invention of easy to use browsers, all supported by exponential increases in computer processing power and computer access in the developed world. Even though the influence of the Internet/Web on business is a very recent phenomenon, it will be shown in this article that the impact has been profound.

The long-term future effect of the Internet/Web cannot be fully understood - the Internet/Web as an event has been written large on a rather small temporal canvas. Perhaps the most important factor to consider when predicting the future impact of the Web/Internet on business, is that it is still in a rapidly evolving state. Other technological innovations, such as the invention of the steam engine which had huge social and economic implications for the Industrial Revolution, do not compare with the Internet/Web in the sense that the steam engine once invented remained very much the same technological artifact, albeit with modifications for greater sophistication. In contrast, the Internet/Web does not behave like an unchanging artifact or thing. The very plasticity of the information and communication technologies that 'drive' the Internet/Web, in combination with the 'nature' of the Internet/Web as a medium, enables a multiplicity of interactions and will probably produce many unforeseen effects.

This article is intended to focus on the impact of the Internet/Web on business. In attempting to do so, one aspect that must be considered is whether it is possible to distinguish between the impact of the Internet/Web from the impact of computers - or broadly IT. The Internet/Web is an extension of and dependent on antecedent IT innovations, making it difficult to separate the two. A similar situation exists with regard to other devices. For example, cellular telephones and personal digital organizers have functions that are independent of the Internet/Web, such as making telephone calls or storing business related data. However, these devices can be connected to the Internet/Web, thereby making them in effect extensions of the Internet/Web. Such devices therefore must be taken into account in a discussion of business related usages of the Internet/Web.

At this point it is necessary to observe that in this article the focus is on the rapid uptake of Internet/Web 'technology' among businesses of the first world/developed economies. It is estimated that only two to three per cent of the world's population has access to computers and that computer access is still overwhelmingly located in the developed world. Low levels of access to ICT in developing countries also seem to be distributed narrowly in a small stratum of the economic and political elite. This implies that those individuals/companies in developing countries who/which have Internet/Web connections are in effect restricted to interacting with businesses that are overwhelmingly located in the developed world. It could be argued therefore that an analysis of the impact of the Web/Internet on business in developing countries will as yet reveal very little change. A counter argument would be that developing countries represent important spheres of research because they will be able to leapfrog technologies instead of trying to catch up. For example, they may jump to cellular telephone technologies rather than adopt older 'line'-based infrastructures. The same could occur in the sphere of Internet/Web innovation.

Finally, it is important to put the size of the Internet/Web economy into perspective. The global Internet population is said to grow from 95.4 million by end of 1998 to an estimated 282 million by 2002. Notwithstanding the future exponential growth predicted, this 
population still represents less than three per cent of the world population (Intelligence 2000:101). Therefore it will be some time before the so-called networked economy 'captures' a significant portion of the world population on-line. This fact should inform or contextualize any discussion of the Internet/Web and raises questions about the interaction between the 'netizens' of the Internet/Web economy and the masses of people who are not 'on-line'. Another aspect that must be noted here is the fact that even though a small fraction of the world's people, about six per cent, are mother tongue speakers of English, nearly 54 per cent of people on the Internet/Web use English. This also makes it the dominant language of business on the Internet/Web. As the large populations of Asia and South America come on line, this dominance may be threatened and the shape of what has been called 'e-culture' may not be dominated entirely by English speakers.

It should be noted that the Internet/Web is strictly speaking not owned by any of the nearly 100 million estimated individual and company users logged on. Therefore, there is practically no company that will market the Internet/Web as its own, but there will be uncountable numbers of companies that will sell the Internet/Web because their products are based on and depend on maximizing Internet/Web-based business. Therefore the Internet/Web itself can be understood as a 'product' that must be sold. For many business players such as INTEL, promoting the Internet/Web will have the effect of enlarging the market for their own products. It is therefore necessary sometimes to separate out Internet/Web marketing and hype from Internet/Web 'facts'. Very strong visions are expounded in this regard. For example the chairman of INTEL, Andy Grove predicted: 'Within five years, all companies will be Internet companies or they won't be companies' (Intelligence 2000:92). The intention here is not to question the prediction. Instead the point to be made is that such statements - aside from their empirical value - have enormous power in promoting the Internet/Web. Grove's statement plays on the fear of business managers that their companies will be either obsolete or non-competitive if they do not buy in to the Internet/Web.

To produce a structured account of the dynamic interaction between the Internet/Web and business, this article is based on the following framework. Firstly, a brief account of the value of the Internet/Web to business is provided. Then some of the 'laws' are identified that characterize business that is conducted over the Internet or 'e-commerce'. These are different from commercial transactions conducted in 'brick and mortar' environments. Thereafter, an attempt is made to identify some of the industries and industrial sectors where e-commerce has had the most impact. The fourth section gives recognition to the Internet/Web as a site of business in and of itself. The last section of this article briefly speculates on possible future trends in the impact of the Internet/Web on business.

\section{Value of the Internet/Web to business}

What are the advantages of the Internet/Web for business? The Internet/Web represents a new medium of and for business. Businesses can conduct transactions through the Web either business to consumer (B to C), or business to business (B to B). Internet/Web based activities can be conducted in addition to - or instead of - direct or brick and mortar types of business. The Internet/Web also enables businesses to revise - sometimes to revolutionize aspects of the business process.

Broadly speaking, the Internet/Web offers businesses the opportunity to increase their presence and accessibility (24 hour convenience), flexibility and response time in meeting client requirements, and to reduce costs at various stages in the business process. Ultimately all of these factors are aimed at leveraging greater competitiveness and as a consequence 
increased sales. It must be noted that the jury is still out on the extent to which Information Technology (IT) produces demonstrable economic gains. However, this critical issue of the contribution of IT and the Internet/Web to economic growth and productivity as debated and researched in the discipline of economics will not be discussed further here.

\section{The new economic laws of the Internet/Web}

The 'new economy' the 'networked economy', the 'Internet economy', which subsumes 'ebusiness', 'e-commerce' and 'e-tailing', has been recognized as a new environment that works according to different 'laws' or rules as the 'old' economy. In the same way as water and air are media that enable humans to engage in some similar but also some uniquely different behaviours, the medium of the Internet produces opportunities and challenges to business people that are different from ordinary non Internet/Web-based business interactions. A number of commentators have attempted to identify the key characteristic of the 'new economy':

- The Internet/Web will experience increasing connectivity. The network effect operates where people-orientated companies and Web sites link together for greater benefit;

- interaction and collaboration costs for businesses will drop, leading to new configurations of businesses;

- access to information will become cheaper and less restricted;

- the new economy is global rather than national or local (globalization of markets for skills, products, services and information);

- in the new economy, corporate size counts less than agility and speed of positioning. There is a shift in emphasis away from assets to intangibles such as information. In other words 'value' is no longer tangible (Meyer 1999);

- the Internet/Web facilitates the business process through compressing time and space and providing potential for 'velocity'. The issue of time and space compression which are critical to understanding the influence of the Internet/Web on human and economic relations in the business field will be raised again later with reference to management decision making, efficiencies and cost reductions;

- successful companies will operate with open standards rather than proprietory systems. By adopting a 'follow the free' approach and building market share, the benefits of ubiquity will be realized (Seybold 1999).

- in accessing information, there is a trade-off between richness and reach, where richness refers to the quality of information received and reach to the number of people exposed to the information;

- controls over price are lost and prices fall subject to the mechanisms of price wars. Free pricing or dynamic pricing will prevail in key sectors;

- since the Internet/Web is a virtual space, the problem of consumer mobility is removed. This means that there are potentially large numbers of consumers who might otherwise never have been clients of a business owing to the fact that they are so distantly located from the business premises. The Internet/Web therefore has the effect of 'compressing' space. However, merely placing all persons on the Internet/Web at one moment in a virtual space where they potentially could be in contact with any other person or business entity is no guarantee for business transactions to take place. The business still has to attract people to the site.

The degree to which Web sites are attractive to the consumer/visitor is of critical importance to a business. The number of visitors to the site can be taken as an index of the success of the site and or the product or service that it purveys. More visits imply greater sales of the product being offered on the site, or potentially greater revenues through advertising sales for 
sites that act as service portals or gateways. The extent of traffic is therefore of interest to all marketing departments that have to make strategic decisions about two issues: first, how effective is Internet/Web marketing in comparison with other media and, second, what proportion of spending should be allocated to Internet/Web spend as opposed to other advertising expenditure. The resolution of this question is made all the more difficult given the conflicting views on how to assess the marketing value of a site according to the number of visits/hits/page impressions, etc. Hence the term 'stickiness' was to refer to the number of times a customer visits a site and the length of time that she/he would stay there. The shortterm future of the Internet/Web as business medium will to some extent be dependent on the confidence marketers have that it is a viable medium to advertise in. Growth in the Internet/Web is dependent on revenues. In the long term, as the Internet/Web expands with more people getting access, marketing departments are likely to feel that they have no option but to ensure that their products are well represented.

\section{Analysis of growth of e-commerce}

An assessment of the impact of the Internet/Web on business must include an analysis of how this utility has changed previous forms of conducting business and how it serves as an environment that produces opportunities for entirely new business forms to emerge. The latter will be the focus of the next section.

The Internet/Web has not influenced all economic sectors evenly for two reasons. First, there are particular business activities that are uniquely suited to or which lend themselves to conversion into Internet/Web-based forms. Secondly, business on the Internet/Web cannot operate efficiently or profitably without a critical mass of consumers or subscribers. The distribution of Internet/Web access on a global scale is so far highly concentrated in certain societies (North America, Europe and the Pacific Rim) (Intelligence 2000:93). Even within these localities with privileged access, in comparison to the rest of the world, access to the Internet/Web on a national level is still highly skewed towards the middle class and more affluent sectors. These social groups, as markets with a greater disposable income and greater propensity for consumption and leisure activity, are the target of Internet/Web businesses.

The problem in assessing the ways in which the Internet/Web has influenced business is that not all companies are purely Internet/Web based, but have significant operational interests in that medium. One way of dealing with this problem is to identify such companies on the basis of what percentage of their total revenues are Internet/Web derived. One recent study based on this criterion (Intelligence 2000 Special Edition) showed that about two thirds of the top 100 companies are not fully Internet/Web based. Although just over one third of these companies primarily are business to business (B to B) companies, their share of revenue is 71 per cent, which provides evidence of the importance of the $\mathrm{B}$ to $\mathrm{B}$ Internet/Web sector.

Other than Internet/Web, ICT and support-related businesses, the number of companies per industry in the top 100 provides an interesting picture of the level of Internet/Web integration. Over 20 per cent of the top 100 Internet/Web companies are in the retail trade, 14 per cent in high tech manufacturing (mostly of computer hardware), 14 were in media/publishing, 13 per cent in financial services, and nine per cent in the travel industry. Interestingly, two entrys in the form of UPS and FedEx entered the rankings giving recognition to the importance of shipping physical goods to customers. In Internet/Web companies, the rankings consist mainly of the following types: net access or portal businesses (e.g. AOL, MCI WorldCom, At Home), retail (e.g. Amazon.com), 
distribution/logistics companies (e.g. Marshall Industries and Ingram), content portals (e.g. Yahoo, Lycos, Excite), content providers (e.g. Getty Images), and online auctions (e.g. Ebay, Ubid).

The discussion now turns to identifying some examples of the impact of the Internet/Web by industry.

A significant locus of Internet/Web activity is media and entertainment. This caters for a market of consumers who have leisure and income. Media and entertainment have also been the target of high tech development where, as a result of advances in the information carrying capacity of the Internet/Web, the 'convergence' of various media has been made possible. As a result, high quality signals (which may include digitized voice, image and other information) can be 'streamed' to consumers on demand. In practical terms however, the content of media and the capacity to deliver have been developed as separate domains, but this is also changing as is evident in the merger of Time Warner and AOL as a concrete example of the coincidence of connectivity, content entertainment and delivery in one corporate entity (Intelligence 1999:34).

The capacity to 'stream' information on the Web/Internet has led to the development of devices that take advantage of this capacity. An example of this is the MP3 digital recorders which can be used to download music off the Internet/Web. The MP3 technology is less remarkable for its technology than for the effects that it will probably have on the prerecorded music industry of CDs. With an MP3 recorder, a client is not obliged to buy a CD containing several songs he/she does not like, but will download any song/performance as his/her choice dictates. Thus, when taken together, the enabling technology of the Internet/Web together with the MP3 technology has the potential to 'kill' the market for CDs by the end of the first decade of the 21st century. This example highlights the rapid changes that a technological innovation can bring if it is coupled with the massive market of the Internet/Web.

However, the tendency to understand the business of the Internet/Web as though it will automatically sweep the whole globe needs to be unpacked so as to understand more clearly the effect on peripheral markets. To take the CD as an example, it will be more accurate to predict that the CD market will be decimated only in contexts where there is a competing technology. What is likely to happen is that the CD manufacturing market will focus on servicing the underdeveloped world where the majority of people do not have access and are unlikely to have access to the Internet/Web for a long time. This represents another example of the dumping or migration of older technologies to poorer parts of the globe.

The Internet/Web is an ideal environment for locating services that are information based. This means that clients can access information at will, at any time of the day and from any place as long as they have a device that can hook them up to the Internet/Web. The attractiveness of web-based information sites is further enhanced by the ease with which such resources can be electronically maintained and updated. As a consequence, there has been a mass migration of businesses that operate databases of various kinds on the Internet. The range of instances is extremely broad, but three will suffice here. The first is companies that provide business management information on economic and financial indicators. The second is businesses that own large-scale reference works like the Oxford Dictionary or encyclopedias that are accessed by subscription. Thirdly, businesses in the financial services sector like banks view the Internet/Web as an ideal medium through which they can sustain or even increase the range and quality of service through enabling clients to conduct business remotely. This produces significant savings to costly person-to-person interactions. Clients can access the database for details on all their accounts and can manipulate their affairs safely and conveniently at any time. 
The Internet/Web lends itself to forcing down costs at the point of sale for several reasons. Firstly, consumers can potentially access considerable volumes of information in comparative shopping to make the best price-value decision. Secondly, clients are very 'mobile' in that they can leave a site and enter another site at the click of a mouse. Thirdly, the Internet/Web produces an environment where theoretically large numbers of businesses compete with each other for theoretically huge consumer markets. Both businesses and consumers can benefit from the competitiveness that is driven by mobility and information access. To keep competitive in a market that is extremely dynamic, businesses are forced to move to dynamic pricing strategies. These forces have been at the heart of the success of Internet/Web auction businesses such as Ebay, which represent very efficient mechanisms for maximizing the numbers of sellers and buyers.

The Internet/Web has also revolutionized the traditional retail sector where traditional 'brick and mortar' storefronts are being supplanted in some cases, or at least supplemented by virtual storefronts. The construction of a virtual storefront on a Web site means that the business concerned can cut down on all costs related to floor space, staffing and stock. The retail areas where Internet/Web storefronts have been particularly successful have been in products that are purchased regularly, such as pet food or cosmetics. However, firms such as Amazon.com have shown phenomenal success in retailing books and CDs.

The advantages of Internet/Web - or basically selling off a computer - have caused some businesses to reassess their approach to conventional retailing. For example, instead of having a full range of commodities, the business can stock only the fastest selling lines and then install a set of computer terminals where customers can access information on all other models/commodities available. In this regard, computers may have a great value in terms of allocation of floor space. |This strategy has only recently become a viable option with the increased sophistication of Web site design applications to make virtual shopping an informative and enjoyable experience.

The increased attention that businesses have given to Internet/Web operations has contributed to changing consumer to business relations. But the goods still have to be delivered to the client. The issue of the supply chain is less problematic for some goods than others. For example, supply chain difficulties occur where goods are perishable, such as food and groceries. This means that even though the Internet/Web presents itself as a viable medium for the consumer to conduct the front end of the transaction, the back end or delivery is a problem on account of the nature of the commodities in question. If not overcome, these conditions will preclude the long-term viability of certain business forms on the Internet/Web.

In the realm of B to B transactions, the Internet/Web has produced profound changes. The first level of influence occurs in respect to the links in the chain of supply between the manufacturer, the supplier and the customer. Traditionally, this supply chain moved through three or more stages: from manufacturer to wholesaler to retailer to the customer. This process was a necessary organizational response to the requirement of distributing huge quantities of mass produced items to the eventual consumer. The medium of the Internet/Web presents the opportunity for manufacturers to deal directly with consumers through developing a Web-presence and designing an efficient delivery system for products. This means that the other intermediaries can be cut out, which will reduce distribution times and also lower costs.

The Internet/Web can also be harnessed to alter the relationship between the process of manufacturing and the final delivery of the product to the consumer. This entails a move away from mass production to what has become known as 'networked production'. The process of 'networked production' is made possible, firstly, through the development of 
flexible post-Fordist production configurations that are possible through the application of IT (such as robotics) to production problems and, secondly, to the medium of the Internet/Web that facilitates information flows between the components in the production process. This contributes to the possibility of creating manufacturing processes that are so 'perfectly' designed as to be virtually 'frictionless'. Furthermore, networked production also entails utilizing the flexibility of the Internet to route information about customer preferences concerning a particular product into the actual production process. This leads to what has been called the 'mass customization' of production.

To use a hypothetical example, a company receives a sales order through its 'shop front'. The sales order, which may even be paid for in advance, includes specifications to suit the exact needs or requirements of the client and the product is then manufactured and delivered. Because the company can make up the product to client specifications, it does not have to keep a huge stock of 'models' to satisfy a range of customer requirements. In addition, because customer requirements in the form of orders are always known in advance of production, there is no need to carry a large stock or inventory of parts/materials at the factory. These can be ordered as needed and therefore are not paid for until they are used. This approach depends on a highly effective and integrated system of communication between the source of the order, the company and the suppliers of raw materials/parts to ensure that the production cycle is short and efficient.

The Internet/Web presents an ideal medium for the construction of 'real-time' networks that essentially facilitate the high speed flows of information that enable the resolution of seemingly intractable complex problems. For example, a major problem for firms in the trucking industry is the problem of matching empty or half empty trucks with loads. This is particularly difficult in a large industry that services frequently random and episodic needs of clients on a complex network of routes. An American company, the National Transport Exchange provides a service that involves networking the databases of many companies to enable a previously unthinkable level of 'freight matching' and as a consequence, produces significant efficiencies for the subscribing companies. As with many such innovations on the Internet/Web, the success of this venture depends on scale to increase benefits (Grebb 1999).

The Internet/Web is having a major impact on the supply chain in various economic sectors. These impacts are taking effect in a number of industries as varied as aircraft and car manufacturing and chemical supplies. By streamlining purchase orders, handling real-time order tracking and providing trend analyses, by maximizing the comprehensiveness of online catalogues of parts/products, and by maximizing their purchase power on account of high numbers of subscribers, such companies acting as 'infomediaries' are able to achieve significant efficiencies and cost savings (Intelligence 1999:39-44).

The kinds of changes sketched here imply the construction of new kinds of organizations that employ the capacity of the Internet/Web medium to provide a single point of contact between a 'community of customers' and a 'community of suppliers'. This is a departure from the pre-Internet patterns which dominantly involved single diadic relationships between clients and suppliers. These new enterprises or 'metamediaries' are likely to flourish in areas such as childbirth, weddings and gardening in the business to consumer (B to C) market and in areas such as logistics management (see trucking example above) and procurement (see the chemicals supply industry example above) in the B to B markets (Sawhney 1999).

Other industries have been shaken by the melting of boundaries between buyers and sellers. Industries affected include the travel, car dealer and newspaper industries. In the travel industry, Internet/Web-based companies provide consumers with a wider range of accessible information as well as convenience with securing reservations. This has two consequences: firstly, it means that less informed travel agents are being displaced, and secondly that, with 
lower overhead costs, Internet-based travel companies are able to cut commissions thereby intensifying competition. The newspaper industry was negatively affected by the arrival of television, and Internet/Web-based interactive and customized newspaper services are set to further erode the base of the paper medium, especially in the small and medium range. Internet/Web-based papers with their rising subscription numbers are also beginning to attract a significant share of classified advertising sales. In the car dealer market, Internet/Web-based businesses are able to undercut dealer costs considerably and increased levels of information access available on the Internet/web has made consumers less tolerant of prices offered by off-line dealers.

The Internet/Web has also begun to shape new business policy on human interactions in the Netspace. The new medium of the Internet/Web represents a new behavioural space. The need to understand how people think, make choices and behave in cyberspace in relation to the products and services offered by companies has become critically important. Web sites are seen as mirrors in cyberspace of the corporate identity of companies. The preoccupation with clients has spawned a new focus on the client or customer as recognized in the growth of the fields of enterprise resource planning and customer relationship management (Intelligence Special Survey 2000:46-68).

In addition, the working lives of millions of employees are likely to be affected in some way as the methods and practices of 'running a business' and managing human resources are shifted by Internet/Web considerations. Growth of the Internet/Web has the effect of shaping sectors in the job market. While many white-collar administrative jobs are becoming redundant as a result of the effect of the Internet/Web on various industries as outlined above, new jobs are also being created. These include job titles such as: 'metrics analyst', 'virtual organization leader', 'content engineer', 'consumer experience manager', 'chief Internet officer' and 'chief knowledge officer'. The occupational profile of economies where the Internet/Web takes hold is likely to reflect these and other new corporate roles (Donohue 1999).

The Internet/Web also is likely to have a significant influence on the demographic structures of business operating environments that have traditionally been based on clumping large numbers of workers together in office blocks. The connectivity advantages of the Internet/Web are likely to lead to greater numbers of workers productively working from home, at lower cost to themselves and to their employers.

Finally, the approach of business to knowledge is changing. Intranets are utilized increasingly as a means of limiting and controlling information deluge and routing, channeling and organizing information. The Internet/Web is placing greater pressure on new approaches to HRD and lifelong education that focuses on ICT as a major source of training content.

\section{Internet/Web as a site that creates new needs and opportunities for new business forms}

The impact that the Internet/Web has on existing business practices and its terrifying capacity to render pre-Internet age business and commercial ventures obsolete have been given some attention. In this section, the Internet/Web is analysed as a relatively new terrain of business that generates increased demand for goods and services, some of which did exist before the Internet age and also for a basket of goods and services that are entirely new.

The most obvious place to start here is in recognizing that the Internet/Web has contributed to the increased demand for computer hardware of all kinds. This has occurred as a result of 
the need for companies to generate an active Web presence and as a result of individuals seeking access to the Internet/Web for private business or recreational purposes. This demand for hardware can range from stand-alone PCs to heavier duty equipment that can support the needs of Internet Service Providers. Demand in this regard is set to increase as the Internet/Web grows. For example, the CEO of Intel Corporation, Craig Barrett cited estimates that only five per cent of the server infrastructure that is needed to accommodate Internet/Web growth over the next five years is in place (Intelligence 2000:30).

The Internet/Web exists as a massive network of humans who in theory are technically capable of interacting with each other. The invention of HTML by Berners-Lee in 1989 enabled business entities to construct virtual environments in which they can 'represent' themselves, their services and their products. These increasingly interactive virtual spaces are built to facilitate sales and customer satisfaction. As a result, a burgeoning industry has grown in the form of businesses that specialize in designing the Web-presence of companies. Some consultants provide a comprehensive service related to the creation of an e-business identity. This can range from consulting, design and editing and development, to process administration, Web hosting, transaction and security. In addition, such consultancies will also advise on and design Web-based customer service applications including so called 'back-end' services such as administration, processing, call centres and even the fulfilment of the transaction in the form of delivery of products. This kind of service is very expensive, but recently the small and medium sized businesses are also being targeted by purveyors of more limited 'shrink-wrapped' options for a Web presence, some provided free by content portals such as Yahoo.com.

The Internet/Web has sparked growth in the development of ever increasingly powerful mobile devices, that is other than computers, that can enable access to the Internet such as mobile telephones and Personal Digital Assistants. From this we can infer that the perceived need for access to the Internet/Web as an information source and active business environment is sufficient to drive the development of devices that can quickly enable an individual to access strategic information from remote sites. In support of such devices, wireless application protocol (WAP) has been developed to enable browsing the Internet/Web from a mobile phone.

Traffic on the Internet/Web has increased exponentially in line with growth in the total number of computers that are linking to the Internet/Web. Traffic has also increased as a direct consequence of the convergence of information and communication technologies. Video, voice, audio and data can now all be processed and transmitted as digital signals 'down the same pipe', but the increasing complexity of such information-rich transmissions as well as requirements for improved signal quality has produced demand for increased carrying capacity - or bandwidth - of the electronic and physical media. In turn this demand is driving the increased layout of physical infrastructure such as the industries responsible for production and installation of optical cable on the one hand and on the other, rapid increases in the number of Western built commercial geo-stationery satellites. The provision of bandwidth as driven by Internet/Web demand has in turn spawned its own mini-industry in the form of what is known as bandwidth exchanges.

The provision of bandwidth is extremely high-tech and expensive, and until recently has been bought by subscription in advance. When such a facility is not used to capacity, this is a loss-making situation to the business that is purchasing the bandwidth. The concept of a bandwidth exchange was developed by entrepreneurs who essentially act as brokers selling and buying 'unused' bandwidth, thereby raising levels of utilization and efficiency and in the process making a profit through taking a percentage on each transaction. The bandwidth exchange is a good example of how an entrepreneur who identifies a market inefficiency can exploit the situation by setting up a virtual shop. 
The Internet/Web has also produced a new generation of companies that exploit the social and economic fabric that underpins the Internet/Web. One example is provided here, namely the business AllAdvantage.com. AllAdvantage targets people who surf the Internet and offers them payment for doing just that. How this works is that people register/sign up with AllAdvantage providing only very basic demographic details (name, postal address, email and age), which are kept confidential. Subscribers agree to download a 'viewbar' or window which occupies a $2.5 \mathrm{~cm}$ high block on the screen of their computer. While the subscriber surfs the Internet/Web, banner-style advertisements are constantly streamed in this window. As the subscriber surfs the net, their viewing habits are analysed and these results are used to develop a profile, which is 'sold' to advertisers. This produces a situation where AllAdvantage can sell advertising space that is targeted to subscribers with specific interest profiles based on analysis of their Internet/Web surfing - while their identity is protected. The kickback for subscribers is that a percentage of the revenues generated by AllAdvantage is paid back to them in exchange for surfing the Internet/Web. The Window streams advertising that coincides with their interests. Subscribers who refer other people who join the scheme earn further income, which makes the AllAdvantage system similar to a pyramid scheme. Essentially the system will work until revenues from advertising drop below the payment per hour promised to subscribers for surfing. This is an example of forms of so called 'viral marketing' which exploits the structure of consumer relationships on the Internet/Web (Batstone 2000).

The dynamics of the Internet/Web produce a situation where information is a critical management resource and critical part of consumer service. As a result, greater proportions of business process information is imported into networked electronic media and, at the same time, more critical information becomes accessible via an Internet/Web presence for clients producing multiple access to a company's repository of 'knowledge'. The strategic paradox of companies in the Internet age is to make more information available that will increase success but, paradoxically, this produces the opposite imperative to control how information is distributed and used. As a consequence, a major field of growth is the Internet/Web-based security industry or more broadly 'risk' management in the Internet environment. In this regard, businesses with an Internet/Web presence invest in a variety of strategies to ward off computer viruses and to forestall hostile attacks from the 'outside'. The central concern here is to secure the networked information systems and databases of a business from loss or degradation. Naturally, there is also a risk of attack from inside the organization by a disgruntled employee, but this article is focusing on the external impact of the Internet on business management.

But this is not the only aspect of concern as companies are also vitally interested in ensuring that productivity of employees is sustained at the highest levels possible. Losses of productive time through employees surfing the Internet/Web for non work-related purposes is considered a significant enough problem for businesses to invest in so-called 'littlebrother' software. Software programmes either limit access to sites that have been pre-identified through an application of a keyword system, or systematically monitor employee surfing behaviour so that employers have evidence with which to confront errant workers. Such forms of software - and variants to protect children - exist entirely as a response to limit or monitor behaviour on the Internet/Web.

So far the issue of security has been viewed in terms of the need to provide protection of the 'information assets' of a business in the form of databases and systems that may be exposed to risk. As critical is the need is protect transactions from observation and possible fraud. The Internet/Web exists primarily as a medium for business interactions, which invariably requires financial transactions. A growth node associated with the Internet/Web has arisen in the form of companies that design software packages/systems - usually based on some form of encryption - to secure communication of confidential financial or other information. Other 
companies specialize in biometric recognition in order to limit access to strategic information.

Another important area of development on the Internet/Web involves the process of payment for goods and services. The lack of a system - or systems - that works well is retarding Internet/Web-based B to C transactions. Essentially there are two problems. Firstly, almost 90 per cent of Web transactions of the B to $C$ type are based on the credit card. Even though virtual credit card-based transactions are considered statistically safer than using a card in a 'real' environment, many potential Internet/Web consumers are not convinced and are keeping their business away. Therefore, the entrepreneurs that devise a secure system of payment which is 'bought' or accepted by significant numbers of buyers and sellers will reap rich rewards. The second problem for businesses on the Internet/Web is so-called micropayments where, for example, a consumer would be willing to pay $\$ 1.50$ to look up a word on the Encyclopedia Britannica but would definitely not be interested in buying an annual $\$ 400$ subscription. Many other examples of a similar kind can be found on the Internet/Web which lends itself to such forms of information selling. But the problem is that there is as yet no universally accepted approach to micro-payments. Again, there appears to be considerable business development work on resolving this problem and the promise of rich pickings to the business entity that finds an appropriate method. These examples suffice to show how the Internet/Web as a commercial environment has generated its own set of problems, which in themselves serve as future areas of development.

These examples suggest that, at least in terms of the Internet/Web, the relative contribution between hardware and software may be shifting towards increased emphasis on developing the software to enable various functions and activities. Put slightly differently, users needs are increasingly driving computer solutions rather than technology and IT (Intelligence 2000:98). An example of this lies in the increasing world-wide focus of CIOs and IT managers on integrating business infrastructural software. This emphasis on enterprise application integration (EAI) is seen as essential in enabling companies to improve or streamline business processes and to achieve higher levels of automation. The latter is frequently expressed in terms of automating the 'front office', the 'back office' and ultimately automating customers - although this is easier said than done (Desmond and Acly 1999).

Finally, this examination of the phenomenal impact of the Internet/Web on business would not be complete without reference to the recent recognition from venture capital that companies whose primary business is concerned with the Internet/Web represent a fertile field of speculation. This interest does not derive altogether from interest only in Internet/Web businesses but must be put in the context of growing emphasis placed by venture capital on technology related investments as offering significant opportunities for growth. Thus Internet/Web related companies have to compete with other technology embedded economic sectors such as the biotechnology industry, and also against other 'tech' firms within the broader IT sector. This is a volatile field of investment activity which has been hurt - though temporarily - by negative sentiments associated with the possible effects of the Y2K bug. More serious concerns have been expressed by some investors over the massive capitalization of so-called 'hollow corporations' in the Internet/Web field many of which as 'knowledge-based' entities deal with intangibles rather than fixed assets.

\section{The Internet/Web business, the state and the legal infrastructure}

The Internet/Web represents a new 'frontier' for the practice of business. The metaphor of the 'frontier' is appropriate here because in colonial times the frontier represented a territory that was beyond the outer limits of the control of government. It was a space where government 
regulations and laws could not be properly policed or applied. What the Internet/Web has done is it has created a virtual territory which is also to a large extent beyond the control of government(s). This is of concern to governments for at least three reasons. Firstly, business transactions on the Internet/Web have proven difficult to track for taxation purposes taxation is taken here to include the widest range of taxation measures, especially duties on imports and exports. The Internet/Web is a medium through which international flows of currency is difficult to control, especially at the level of transactions as conducted by private individuals. These are very serious problems because government depends on its tax base to pay for public expenditure on utilities, infrastructural development and on social security. The Internet/Web as an international realm that does not recognize national boundaries produces problems for government control. Second, governments are also concerned to protect their citizens from possible harmful effects arising out of conducting transactions on the Internet/Web. There are businesses on the Internet/Web whose activities could be deemed financially exploitative or could prove to be harmful to individual health, and security - including privacy. Broader negative effects cited include the contribution of the Internet/Web to social dislocation. So, governments will seek to safeguard the rights of citizens and of national economies against potential harm.

Opponents of government regulation of the Internet/Web strongly argue that any such intervention by government will only serve to impede growth in the volume of business that is transacted. This begs the question whether any government has the capacity to comprehensively regulate the Internet/Web anyway. Ultimately, governments are bound to protect their - and their citizens' - interests. In contrast, the Internet/Web is essentially an international space requiring perhaps the construction of an international convention that regulates large areas of Internet/Web behaviour such as financial transactions but not cultural issues. These questions cannot be adequately addressed here. They are nevertheless raised as concerns that will inform the future of business on the Internet/Web.

The question of regulation cannot be taken as separate from the law as it relates to the Internet/Web. All government regulations/laws have to be supported by and justified in terms of pre-existing more fundamental sets of law - such as the constitution. The field of Internet/Web law - or 'cyberlaw' - is still developing but not fast enough. This is in itself a major problem because legal systems are slow in maturing - the system of Roman-Dutch law, which is the bedrock of the South African legal system, is the product of hundreds of years of development. For instance, fundamental questions have not been satisfactorily resolved concerning the use of electronic documents as evidence in legal processes. Given that many electronic transactions over the Internet/Web are never printed in a hard copy, this raises questions as to what is an 'original' copy of an agreement or transaction? Issues such as this are likely to critically affect some businesses causing failure or the retardation of their growth if legal battles are not resolved. More cautious individuals and companies are likely to stay away from conducting certain critical business functions on the Internet/Web, but there will also be counter pressure for individuals and companies to be present on the Internet/Web in spite of the pitfalls. The common assumption which seems uppermost is that the opportunity costs of not getting involved will be far greater than the losses resulting from waiting for everything to be ironed out first.

\section{Conclusion}

The Internet/Web has been shown to have had a significant effect on the business world, both in terms of revolutionizing old business practices and sectors and generating entirely new fields of enterprise. 


\section{References}

Barrett, C.R. 2000. Welcome new players on the Net. Intelligence 6(1):28-30.

Batstone, D. 2000. Add it up. Intelligence (6)3:56-60.

Cross, K. (ed.) 2000. Who's Online? Intelligence 6(3):101.

Cross, K. (ed.) 2000. Are SA companies ready for ecommerce? Intelligence 6(4):92.

Cross, K. (ed.) 2000. Who's Online? Intelligence 6(4):93.

Davis, J. (ed.) 1999. Are you next? 20 Industries that must change. Intelligence 5(7):39-44.

Desmond, J.P. and Acly, E. 1999. Beating the integration blues. Software Magazine 19

(2):29-33

Donohue, S. 1999. New jobs for the new economy. Intelligence 5(9):42-49.

Grebb, M. 1999. Wise load. Intelligence 5(8):28-32.

Intelligence. 2000. Editorial: Are you next? 20 Industries that must change. 5(7):39-44.

Intelligence. 2000. Editorial: Top 100 companies at a glance. Special edition 6(1):74-105.

Intelligence. 2000. ERP to CRM. Special survey 5(10):46-68.

Intelligence Special Survey. 1999. Software development continues. Intelligence 5(8):98.

Lawlor, P. 2000. 2000. Arrives at pace. Intelligence 6(3):32-36.

Meyer, C. 1999. What's the matter? Intelligence 5(8):67-70.

Sawhney, M. 1999. Making new markets. Intelligence 5(9):58-63.

Seybold, P. 1999. Ubiquity breeds wealth. Intelligence 5(7):53-59.

\section{Reference works}

Allwords.com http://www.allwords.com

Dictionary.com http://www.dictionary.com

InstantWeb.com http://www.instantweb.com

Merriam-Webster http://www.M-W.COM

Thesaurus.com http://www.thesaurus.com

Wordsmyth.net http://www.wordsmyth.net

\section{Disclaimer}

Articles published in SAJIM are the opinions of the authors and do not necessarily reflect the opinion of the Editor, Board, Publisher, Webmaster or the Rand Afrikaans University. The user hereby waives any claim he/she/they may have or acquire against the publisher, its suppliers, licensees and sub licensees and indemnifies all said persons from any claims, lawsuits, proceedings, costs, special, incidental, consequential or indirect damages, including damages for loss of profits, loss of business or downtime arising out of or relating to the user's use of the Website. 
ISSN 1560-683

Published by InterWord Communications for the Centre for Research in Web-based Applications, Rand Afrikaans University 